\title{
An Approach towards designing of Car Troubleshooting Expert System
}

\author{
Ms. Neeta Verma \\ A.P (CSE) \\ IPEC, UPTU \\ Ghaziabad, India \\ Rashi Aggarwal \\ B.Tech (CS, 7th Sem) \\ IPEC, UPTU \\ Ghaziabad, India
}

\author{
Yash Jindal \\ B.Tech (CS, 7th Sem) \\ IPEC, UPTU \\ Ghaziabad, India
Swati Jain
B.tech (CS, 7th Sem)
IPEC, UPTU
Ghaziabad, India

\begin{abstract}
Car problem detection is a complicated process which demands high level of knowledge and skills. Our aim is to develop an expert system on car maintenance and troubleshooting that is capable of assisting car's owner in dealing with their car problems and troubleshooting them whenever the time is limit and the human expert, also known as mechanics is not available at that very point of time. This paper provides affective design issues concerning the problems while driving a car and will give a logical solution which would help in rectifying those problems. The system would contain various set of rules for detecting different type of failures which can be easily handled by the driver and will give their causes. Here the system mainly deals with the starting problems of car and detecting various other large scale problems.
\end{abstract}

\section{Categories and Subject Descriptors}

[Computer Science/ Information Technology]: Artificial Intelligence-An Approach towards designing of $\mathrm{Car}$ Troubleshooting Expert System

\section{General Terms}

This paper deals with the design issues while creating an expert system; in this case- A car failure diagnose Expert System. This system would be highly reliable for detecting different problems related to car as well as it would be quite economical.

\section{Keywords}

Expert system, car failure diagnosis, knowledge-based system, inference engine, component, prolog

\section{INTRODUCTION}

An expert system can be defined as "an intelligent computer program that uses knowledge and inference procedures to solve problems that are difficult enough to require significant human expertise for their solutions" [1]. We can infer from this definition that expertise can be transferred from a human to a computer and then stored in the computer in a suitable form that users can call upon the computer for specific advice as needed.
Then the system can make inferences and arrive at a specific conclusion to give advices and explains, if necessary, the logic behind the advice.

ES provide powerful and flexible means for obtaining solutions to a variety of problems that often cannot be dealt with by other, more traditional and orthodox methods [2]. Some of expert system important applications include the following: medical treatment, engineering failure analysis, decision support, knowledge representation, climate forecasting, decision making and learning, and chemical process controlling.

\subsection{Starting with the Problem}

Having a car can be an exciting moment to everyone and they live easily throughout their daily life, mobilizing on the car. But at the same time, each of those who own a car definitely don't want to have a problem on their cars as the troubleshooting of the car can be a nightmare to them especially, women. So, this expert system is hoped to help those who are in need of guides to deal with their car's problems. Although it might not give a complete guides and help as a human expert namely mechanics do, but at least the expert system can give a temporary assistance to those who are in need of an instance help. The system will:

- help the car's owner in making decision on action they should take in their attempt to troubleshoot the car's problem.

- Save much time, cost and energy for the user especially to those who are in hurry as they don't have to wait for the mechanics to repair the damages.

- To get the peoples even more closer the world of computerization and technology.

Besides that, the system will also give the developer an opportunity to get involve directly into the development of expert system, and at the same time help to build the programming skill, as well as problem solving capabilities. 


\section{COMPONENTS OF EXPERT SYSTEM}

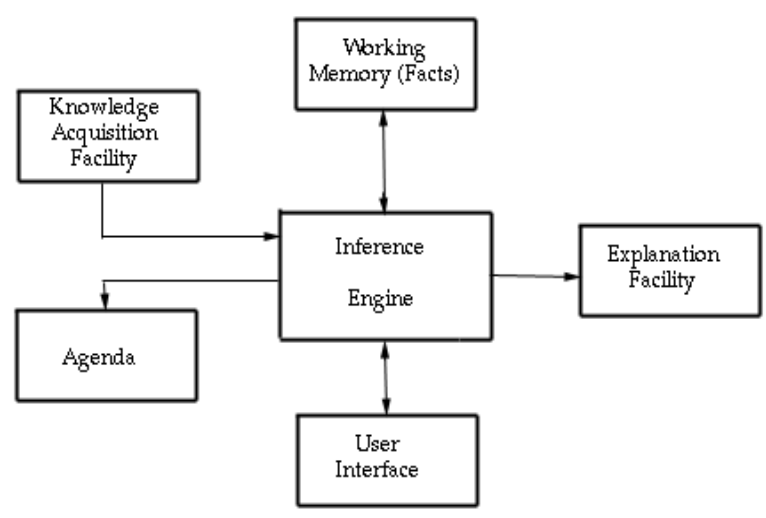

Figure 1. Components of expert system

\subsection{The User Interface}

The user interface is the means of communication between a user and the expert systems problem-solving processes. A good expert system should have an efficient interface. The user interface in this system will be able to accept the instructions in a form that the user enters and translate them into working instructions for rest of the system. The user will be asked several questions which he would have to answer, subsequently a solution will be displayed thereby. Careful attention should be given to the screen design in order to make the expert system appear friendly to the user [3].

\subsection{The Knowledge Base}

Knowledge base is used to store the knowledge of experts, when expert system is reasoning, it is to acquire knowledge and rules from the knowledge base, and repeat matching process of fault conditions, but in the high requirements of real-time inference situations, it needs a new design model to fill the requirements of high speed and stability.

\subsection{The Inference Engine}

An inference engine is a computer program that tries to derive answers from a knowledge base. It is the "brain" that expert systems employ to reason about information in the knowledge for the ultimate purpose of formulating new conclusions [4]. We can say that the inference engine is the program that locates the appropriate knowledge in the knowledge base and infers new knowledge by applying logical processing and problem-solving strategies.

\subsection{The Knowledge Acquisition}

Knowledge acquisition is a method of learning, first proposed by Aristotle. Knowledge acquisition includes the elicitation, collection, analysis, modeling and validation of knowledge for knowledge engineering and knowledge management projects [5]. The knowledge in this system has been collected through the following means:
- Through car service stations

- Through workshops

- $\quad$ Through garage

- Through car mechanics

- $\quad$ Through petrol pumps

\subsection{Explanation Facility}

Illustrates to the user how and why the system gave a certain cause for the failure, i.e. explains the reasoning of the specific car problem to the user.

\section{RULE-BASED SYSTEM}

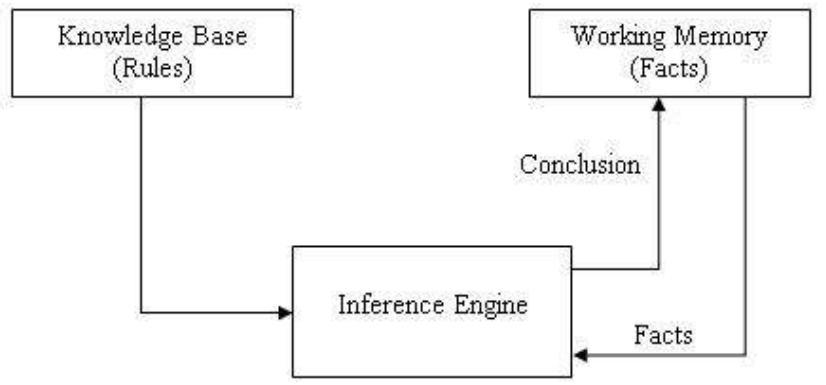

Figure 2. Rule-based expert system model

The Knowledge Engineer acquires knowledge from experts in specific field, through standardization process, and sorts out the collected knowledge from experts to formed rules. In Car diagnosis system, the way of knowledge representation is production rule. The production rule uses the presentation way of "IF P THEN Q", P is precondition, and Q is conclusion [6].For this system, some of the rules can be:

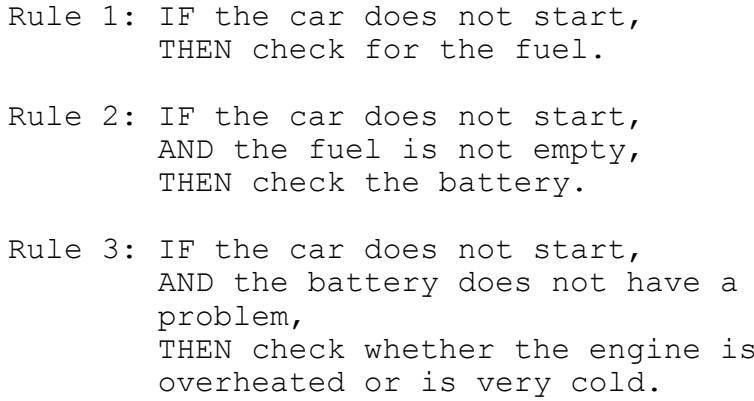

Figure 3. Three assumed rules in the agenda.

\section{EXPERT SYSTEM USING PROLOG}

Prolog has some significant assets that aid in the development of expert systems. The existing facilities of Prolog and its easy availability make it very suitable for the rapid prototyping of certain kinds of small-to-medium scale systems [7]. The basic features of Prolog that aid the design of expert systems are: 
- Support for search-based computation via backward chaining,

- The ability to have suspended variable bindings via the logical variable,

- Use of (syntactic) unification as a basic pattern matching technique to support a clause-based style of programming, and

- Simple and elegant semantics that offer a cohesive framework for rule-based systems, and which allow the formal manipulation of logic programs.

The following are certain rules written in prolog which are used in this system:

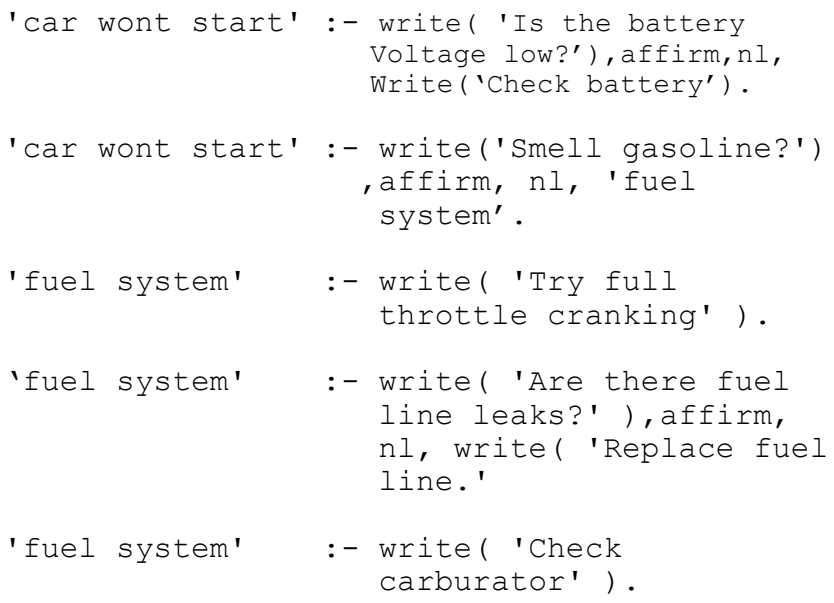

\section{CONCLUSION}

It is not denied that the role of expert system in almost every part of human's life is getting bigger. This is all because of the performance that the expert system has shown of what it can do to help the human beings. The expert system may be useful to execute the routine works and let the human expert to do the rest especially the more difficult jobs. This Expert System for Car Maintenance and Troubleshooting is believed to be the right and excellent idea as another application of expert system and how we can utilize its enormous functions.

\section{ACKNOWLEDGMENTS}

Our thanks to ACM SIGCHI for allowing us to modify templates they had developed. This research was supported by the director and the faculty of Inderprastha Engineering College, of UP technical University, India.

\section{REFERENCES}

[1] Joseph Giarratano Gary Riley (2004). Expert Systems: Principles and Programming, Fourth Edition.

[2] Shu-Hsien Liao (2005). Expert system methodologies and applications -a decade review from 1995 to 2004, Expert Systems with Applications, 28, 93-103.

[3] year12ipt.ash.com/untitled-7.html:Components of Expert system

[4] Wu Jinpei, Xiao Jianhua, Intelligent Faults Diagnosis and Expert System. Science Technology Press. 1997.9

[5] www.epistemics.co.uk/Notes/63-0-0.htm:Knowledge Acquisition

[6] GuanHuiLing, HanJie, Principle and Practice of Facility Diagnosis Expert System, 1st, China Science Publishing House: BeiJing, 2000.11, pp5-7.

[7] E. Y. Shapiro, "Systems programming in concurrent Prolog," in Proc. ACM Symp. Principles of Program. Lang., Jan. 1984. 